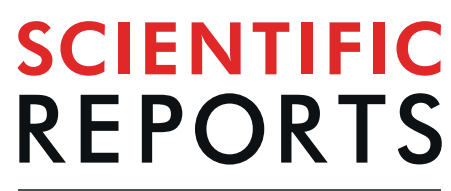

natureresearch

\title{
Global translational repression induced by iron deficiency in yeast depends on the Gcn2/elF $2 \alpha$ pathway
}

\author{
Antonia María Romero ${ }^{1,4}$, Lucía Ramos-Alonso ${ }^{1,4}$, Paula Alepuz ${ }^{2,3}$, Sergi Puig ${ }^{1 *}$ \& \\ María Teresa Martínez-Pastor ${ }^{2 *}$
}

Iron is an essential element for all eukaryotic organisms because it participates as a redox active cofactor in a wide range of biological processes, including protein synthesis. Translation is probably the most energy consuming process in cells. Therefore, one of the initial responses of eukaryotic cells to stress or nutrient limitation is the arrest of mRNA translation. In first instance, the budding yeast Saccharomyces cerevisiae responds to iron deficiency by activating iron acquisition and remodeling cellular metabolism in order to prioritize essential over non-essential iron-dependent processes. We have determined that, despite a global decrease in transcription, mRNA translation is actively maintained during a short-term exposure to iron scarcity. However, a more severe iron deficiency condition induces a global repression of translation. Our results indicate that the Gcn2-elF2 $\alpha$ pathway limits general translation at its initiation step during iron deficiency. This bulk translational inhibition depends on the uncharged tRNA sensing Gcn1-Gcn20 complex. The involvement of the Gcn2-elF2 $\alpha$ pathway in the response to iron deficiency highlights its central role in the eukaryotic response to stress or nutritional deprivation, which is conserved from yeast to mammals.

With the only known exception of some bacterial species ${ }^{1,2}$ the vast majority of living organisms depend on iron as an essential redox cofactor in fundamental cellular processes, including DNA replication and repair, mRNA translation, cellular respiration, photosynthesis and oxygen transport. Although iron is quite abundant in the Earth's crust, its bioavailability is highly restricted under aerobic conditions because of the extremely low solubility of ferric iron at physiological $\mathrm{pH}$. Thus, iron deficiency has become a widespread nutritional problem with important health and economic consequences. Therefore, the acquisition and maintenance of sufficient iron levels suppose a challenge for living organisms, which have evolved sophisticated mechanisms to modulate iron homeostasis.

The post-transcriptional regulation of gene expression plays an important role in the control of iron homeostasis in many organisms, ranging from bacteria to mammals ${ }^{3-5}$. In bacteria, small non-coding RNAs affect RNA stability of genes involved in iron metabolism, storage and photosynthesis ${ }^{3,4}$. In mammals, the binding of the Iron Regulatory Proteins IRP1 and IRP2 to Iron Responsive Elements (IRE) in the $5^{\prime}$ - or $3^{\prime}$-untranslated region (UTR) of mRNAs involved in iron metabolism regulate either stability or translation ${ }^{5}$. The budding yeast Saccharomyces cerevisiae has proved to be a reliable model to characterize the mechanisms of adaptation to iron deficiency in eukaryotic cells. In S. cerevisiae, the Cth2-mediated decay and translational repression of specific mRNAs implicated in iron metabolism allows a metabolic remodeling that prioritizes essential over non-essential iron-dependent processes ${ }^{6-8}$. The mammalian Cth2 homfolog, called tristetraprolin (TTP), also post-transcriptionally modulates the expression of iron metabolism related genes, such as the transferrin receptor and genes within the mitochondrial electron transport chain, to enhance cell survival and maintain cardiac

\footnotetext{
${ }^{1}$ Departamento de Biotecnología, Instituto de Agroquímica y Tecnología de Alimentos (IATA), Consejo Superior de Investigaciones Científicas (CSIC), Catedrático Agustín Escardino 7, E-46980, Paterna, Valencia, Spain. ${ }^{2}$ Departamento de Bioquímica y Biología Molecular, Universitat de València, Doctor Moliner 50, E-46100, Burjassot, Valencia, Spain. ${ }^{3}$ ERI Biotecmed, Universitat de València, Doctor Moliner 50, E-46100, Burjassot, Valencia, Spain. ${ }^{4}$ These authors contributed equally: Antonia María Romero and Lucía Ramos-Alonso. *email: spuig@iata.csic.es; maria.teresa.martinez@uv.es
} 
function under iron deficiency ${ }^{9,10}$. Most of the hitherto described post-transcriptional mechanisms that regulate iron homeostasis involve the regulation of mRNA stability, and in some cases also translation of specific mRNAs. However, little attention has been paid to the effects of iron deficiency on global translation.

Protein synthesis is possibly the most energy consuming process in the cell ${ }^{11,12}$. Thus, one of the primary responses of living cells to nutrient limitation or exposure to stress conditions is a general arrest of mRNA translation ${ }^{13-18}$. Thus, environmental cues including heat shock, oxidative and osmotic stresses, and nutritional deficiencies such as glucose or amino acid limitation, have been described to repress bulk translation ${ }^{13,14,18,19}$. The principal regulation of eukaryotic translation occurs at the initiation phase ${ }^{20-24}$. Under optimal growth conditions, eukaryotic translation initiates with the assembly of the ternary complex formed by translation initiation factor eIF2, GTP and methionyl-tRNA. At the end of the initiation step, GTP hydrolyzes into GDP and the ternary complex is disassembled. Then, the guanine nucleotide exchange factor eIF2B catalyzes the recycling of eIF2-GDP into eIF2-GTP. Upon amino acid deprivation or exposure to different stress conditions, Gcn2 protein kinase phosphorylates the alpha subunit of eIF2 (eIF2 $\alpha$ ), converting eIF2 into an eIF2B inhibitor, which causes a decrease in the levels of ternary complex and a block in global $5^{\prime}$ cap-dependent translation initiation. Interestingly, low ternary complex abundance enhances the translation of the GCN4 mRNA, which encodes for a transcriptional activator of amino acid biosynthetic genes, via short upstream open reading frames (uORFs) ${ }^{16}$. The activation of Gcn 2 kinase in response to amino acid starvation occurs through binding of uncharged tRNAs to its histidyl-tRNA synthetase (HisRS)-like domain, a process that involves the Gcn1-Gen20 protein complex, which facilitates the transfer of the uncharged tRNA from the ribosome to $\mathrm{Gcn} 2^{25,26}$.

Despite that the biosynthesis of multiple amino acids including leucine, lysine and methionine depends on iron, and that ribosome biogenesis and recycling requires the essential and conserved iron-containing protein Rli1, no evidence has reported yet whether protein synthesis is altered in iron-deficient yeast cells. In this work, we analyze the state of bulk translation during the progress of iron starvation in S. cerevisiae. Our results indicate that yeast cells activate a global repression of protein synthesis after exposure to iron starvation. This response is mediated by the Gcn2/eIF2 $\alpha$ pathway and requires the uncharged tRNA sensing complex Gcn1-Gcn20.

\section{Results}

The efficiency of global mRNA translation decreases in response to iron limitation. To investigate whether iron deprivation alters the efficiency of bulk mRNA translation, we determined the polysome profile of wild-type W303 yeast cells cultivated in iron-sufficient $(+\mathrm{Fe})$ or iron-deficient conditions $(-\mathrm{Fe})$, achieved by the addition of $100 \mu \mathrm{M}$ of the $\mathrm{Fe}^{2+}$-specific chelator bathophenanthroline disulfonic acid (BPS). Only a slight decrease in polysome abundance was observed after 3 hours of iron deficiency, compared to 3 hours in iron sufficiency (Fig. 1A,C,E for polysomes/80 S (P/M) ratio). More important, after 6 hours of iron depletion, we observed a significant increase of the monosomal $80 \mathrm{~S}$ ribosome peak and a large decrease in polysome abundance (Fig. 1D,E), compared to the polysome profile obtained in cells incubated the same hours under Fe replete conditions (Fig. 1B,E). These observations are indicative of a general repression of translation initiation during the progress of iron deficiency.

The translational state of specific transcripts is differentially affected under iron deficiency. To analyze how the global repression of translation observed under iron-deficient conditions affected specific transcripts, we studied the distribution of different mRNAs on the polysome profiles, which is indicative of their translational state. For this purpose, we extracted total RNA from the different polysomal fractions and determined the levels of some transcripts by RT-qPCR. Firstly, we analyzed the pattern of two mRNAs encoding for ribosomal proteins (RP), RPS16B and RPL3. We observed that both RP transcripts increased their association to the monosomal $80 \mathrm{~S}$ peak (fraction 4) along with the progression of the iron deficiency (Fig. 2A,B). Regarding the polyribosomal section of the profile, the RP transcript association either decreased (RPL3) and/or shifted to lighter polysomes (RPS16B). Both traits are characteristic of a decrease in the translation level of these mRNAs, which is in accordance to the global translational repression observed upon iron starvation. Secondly, we studied the translation pattern of the housekeeping gene actin (ACT1), which showed the typical profile of a highly translated mRNA under iron replete conditions (Fig. 2C). The ACT1 polysomal pattern was mostly unaffected by the fluctuations in iron availability, and only a slight shift to lighter polysomal fractions was observed after 6 hours of iron depletion. Finally, we examined the distribution of the GCN4 mRNA, which translation is activated when eIF $2 \alpha$ is phosphorylated and eIF2 function is limited ${ }^{16}$. We observed that, under iron sufficiency, GCN4 transcript accumulated in the monosomal fractions, a pattern that was still conserved during mild iron deficiency conditions (Fig. 2D). Remarkably, after 6 hours of iron deprivation, the GCN4 mRNA shifted to the polysomal fractions, which evidenced ribosome enrichment and suggested an enhancement in its translation efficiency (Fig. 2D). To further address this issue, we determined $\beta$-galactosidase activity from wild-type cells expressing a GCN4-lacZ reporter construct containing the four uORFs present in the $5^{\prime}$-leader sequence of GCN4 mRNA (p180 plasmid $)^{27}$. After cultivating these cells in $+\mathrm{Fe}$ and $-\mathrm{Fe}$ conditions, we observed a slight, but significant, increase in $\beta$-galactosidase activity upon iron depletion (Fig. $2 \mathrm{E}$ ). However, the rise in $\beta$-galactosidase activity achieved by iron depletion was not as substantial as the increase observed for 3-aminotriazol (3-AT) control treatment, which limits histidine biosynthesis leading to amino acid starvation (Fig. 2E). Taken together, these results suggest that, despite the global repression of translation that occurs when iron bioavailability is limited, the translation of specific mRNAs is differentially regulated under iron deficiency.

The elF2 $\alpha-G c n 2$ pathway is involved in the global translational arrest that occurs under iron deficiency. Our observation that the translation of GCN4 mRNA increases under iron deficiency suggested that the Gcn2-eIF2 $\alpha$ pathway, which regulates translation initiation in eukaryotes, could be involved in the repression of translation under low iron. Moreover, the increase in the $80 \mathrm{~S}$ peak that we observe under a prolonged iron 
A

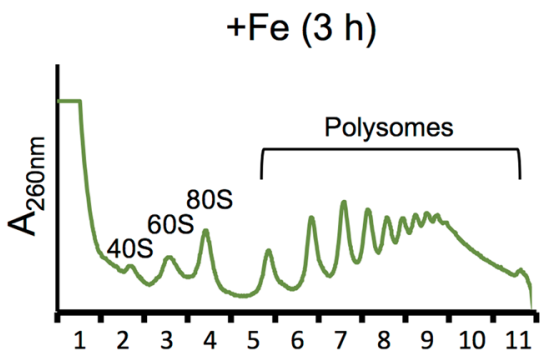

Polysomal fractions

C

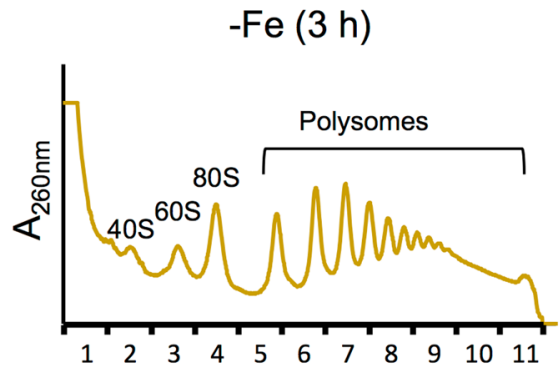

Polysomal fractions
B

$+\mathrm{Fe}(6 \mathrm{~h})$

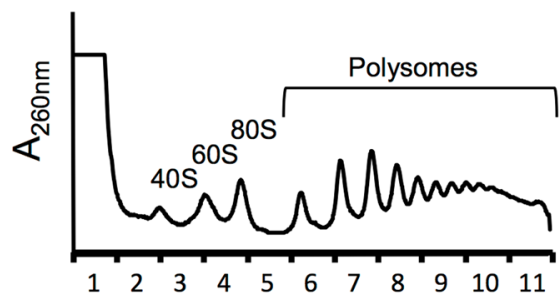

Polysomal fractions

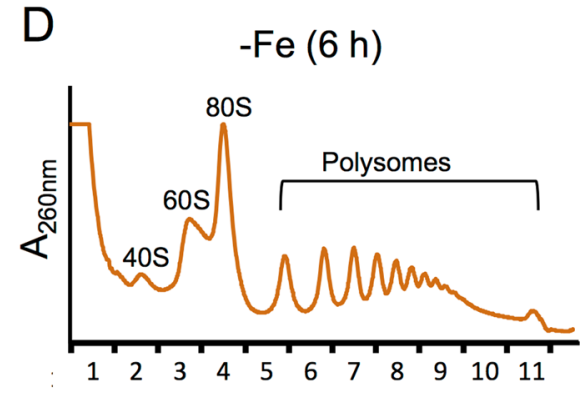

Polysomal fractions

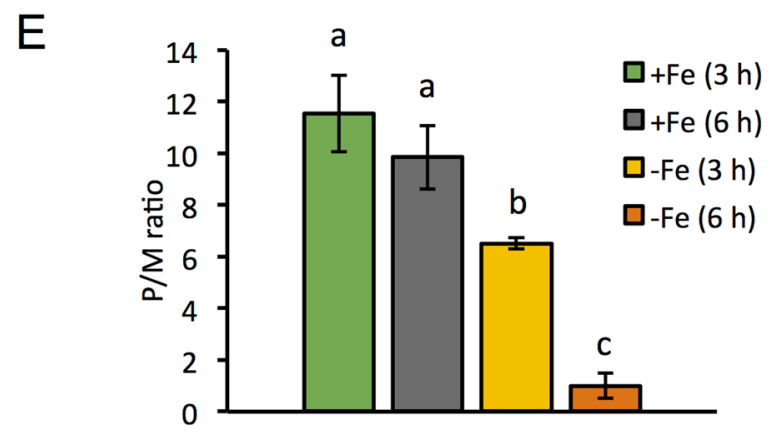

Figure 1. A general repression of translation occurs in response to iron deficiency. Polysome profiles were obtained from wild-type W303 strain cultivated in synthetic complete (SC) medium for at least 15 hours to reach early exponential phase $\left(\mathrm{OD}_{600}=0.2\right)$, and then cells were further maintained in $\mathrm{SC}(+\mathrm{Fe})(\mathbf{A}, \mathbf{B})$ or in $\mathrm{SC}+100 \mu \mathrm{M}$ BPS $(-\mathrm{Fe})(\mathbf{C}, \mathbf{D})$ for $3(\mathbf{A}, \mathbf{C})$ and 6 hours $(\mathbf{B}, \mathbf{D})$ as described in Methods. The $\mathrm{A}_{260 \mathrm{~nm}}$ profiles after gradient fractionation are shown and the ribosomal subunits ( $40 \mathrm{~S}$ and $60 \mathrm{~S})$, monosomes $(80 \mathrm{~S})$ and polysomes are indicated. Three biologically independent replicates were performed in each case, and a representative profile is shown. (E) The average polysomes/monosome $80 \mathrm{~S}$ ratio $(\mathrm{P} / \mathrm{M})$ is represented with its corresponding standard deviation. Different letters over the bars indicate statistically significant differences $(p$-value $<0.05)$.

deficiency also suggested that translational repression is occurring at the initiation stage, which is the main point for eukaryotic translational control ${ }^{20-24}$. Under optimal growth conditions, eIF2 properly initiates translation. However, upon amino acid starvation or exposure to different stress conditions, the Gcn 2 kinase is activated and phosphorylates eIF $2 \alpha$, which interferes with $5^{\prime}$ cap-dependent mRNA translation ${ }^{19,28-30}$. Consistent with this, we observed that the expression of the GCN4-lacZ reporter construct was not up-regulated by amino acid starvation (3-AT treatment) or upon iron depletion in a gcn $2 \Delta$ mutant (Fig. 2E). To further ascertain whether eIF $2 \alpha$ was involved in the translational repression triggered by iron deficiency, we determined its phosphorylation state. By using an antibody that specifically recognizes the phosphorylated form of eIF $2 \alpha$ and another antibody that binds to all eIF $2 \alpha$ forms, we could determine that iron depletion promotes eIF $2 \alpha$ phosphorylation without altering its total protein levels (Fig. 3A,B; Supplementary Fig. S1). These observations point to eIF2 $\alpha$ phosphorylation as a mechanism to control protein synthesis when iron is scarce.

Previous studies have shown that Gcn 2 kinase specifically phosphorylates eIF2 $\alpha$ serine 51 to inhibit protein synthesis in response to amino acid limitation and other stresses ${ }^{19,31}$. Therefore, we analyzed the polysome profile of a SUI2-S51A mutant strain (RS-88), which expresses a non-phosphorylatable form of eIF $2 \alpha^{32}$. Under iron-sufficient conditions, we observed that the polysomes to monosome $80 \mathrm{~S}(\mathrm{P} / \mathrm{M})$ ratio of the wild-type 

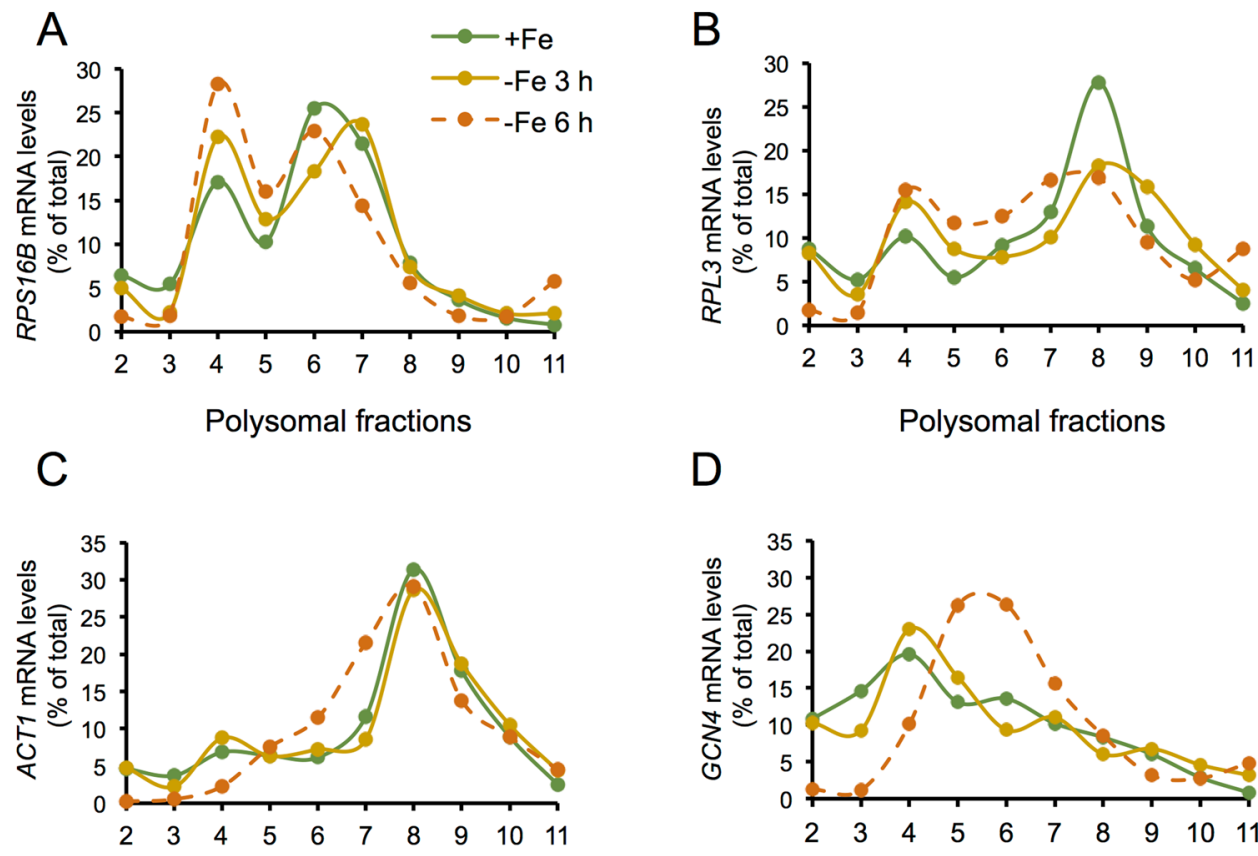

D
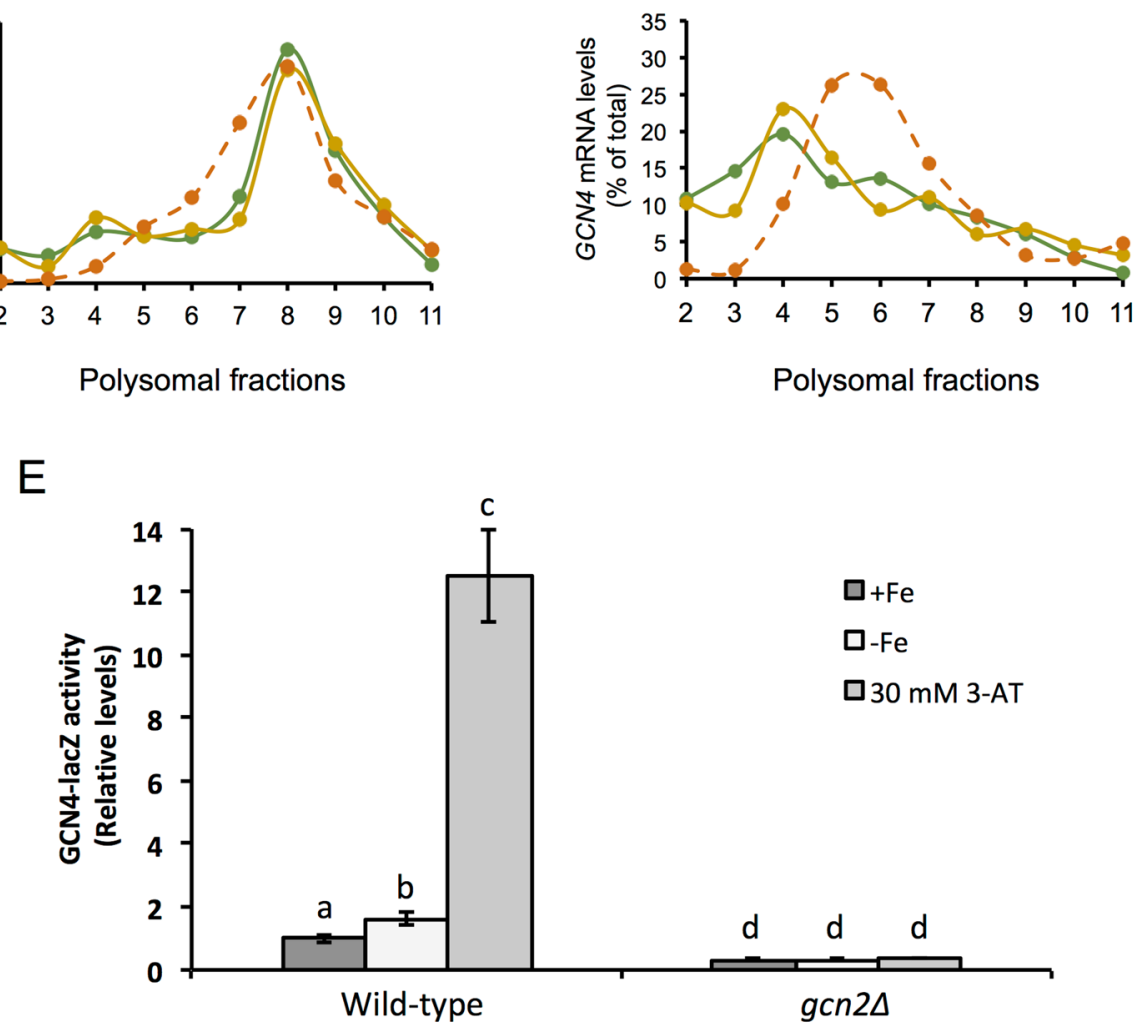

Figure 2. Specific mRNAs are differentially translated in response to iron deficiency. The RNA from individual fractions of the polyribosome profiles shown in Fig. 1 was extracted and the mRNA levels of RPS16B (A), RPL3 (B), ACT1 (C) and GCN4 (D) were analyzed by RT-qPCR as described in Methods. (E) W303 ura3 $\Delta$ and W303 ura $3 g c n 2 \Delta$ cells transformed with a GCN4-lacZ reporter (p180) plasmid ${ }^{27}$ were cultivated in SD $(+\mathrm{Fe}), \mathrm{SD}$ with $100 \mu \mathrm{M}$ BPS $(-\mathrm{Fe})$ and 3-AT, as indicated in Methods. Cells were harvested for $ß$-galactosidase activity assay and the values relative to wild-type $G C N 2+$ Fe cells represented. Mean values and standard deviations from three biologically independent experiments are shown. Different letters over the bars indicate statistically significant differences $(p$-value $<0.05)$.

(RS-86) and SUI2-S51A mutant strains was similar (Fig. 3C,E,G). Consistent with our hypothesis, in response to iron deficiency the P/M ratio lowered in the RS-86 wild-type strain (Fig. 3D,G), although this response was not as severe as the drop observed in the case of the W303 background (Fig. 1). Importantly, the P/M ratio observed for the SUI2-S51A mutant strain did not significantly decrease when iron bioavailability was limited (Fig. 3E-G). From these results we conclude that the phosphorylation of eIF $2 \alpha$ at its serine 51 is relevant for the repression of bulk translation under iron deficiency.

Since Gcn 2 is the kinase that phosphorylates eIF2 $\alpha$ at serine 51 , we postulated that Gcn 2 could be repressing protein synthesis in response to iron deficiency. As expected, the gcn $2 \Delta$ strain displayed a growth defect in media containing 3-AT as well as a defect in eIF2 $\alpha$ phosphorylation (Supplementary Figs. S2 and S3). Then, we determined the polysome profiles of a wild-type yeast strain and a $g c n 2 \Delta$ mutant under either iron-sufficient or iron-deficient conditions. While no significant differences were observed in the polysomal profiles obtained under iron replete conditions (Fig. 4A,C,E), a significant decrease in the P/M ratio was observed under iron starvation for the wild-type strain as compared to the $g c n 2 \Delta$ mutant (Fig. 4). These results evidence that the protein 
A

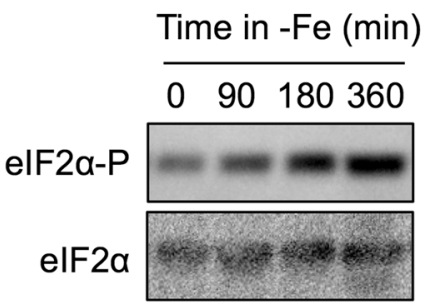

C

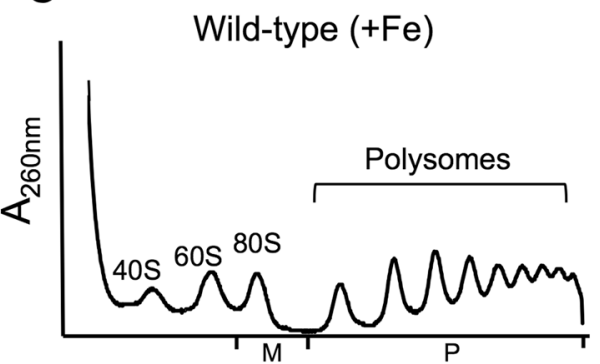

[Sucrose]

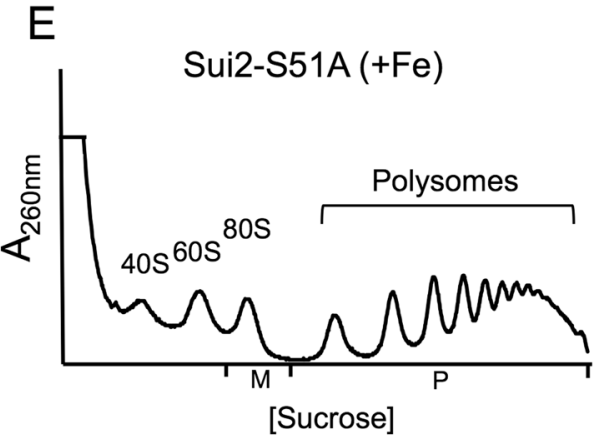

G

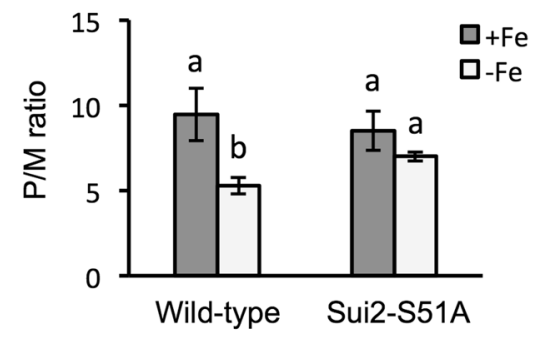

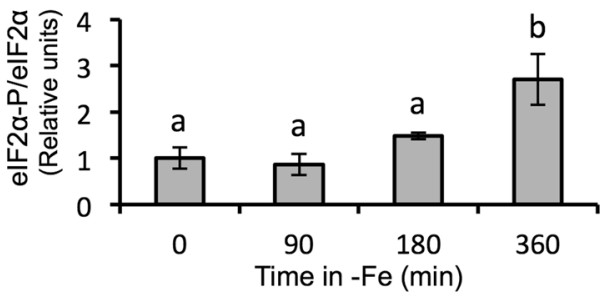

D

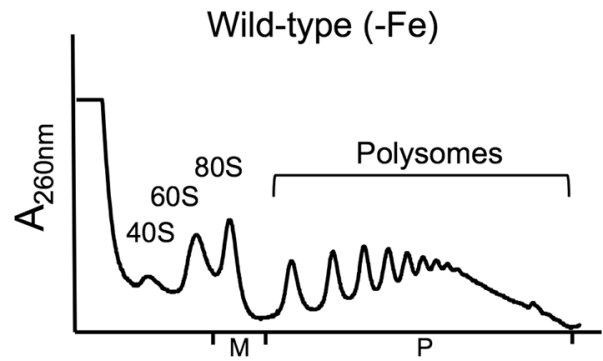

[Sucrose]

$\mathrm{F}$

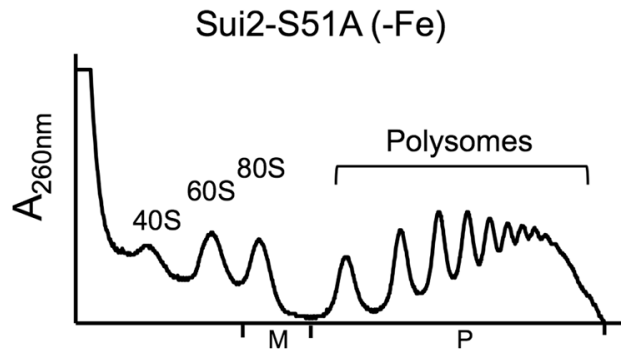

[Sucrose] response to iron deficiency. (A) Determination of eIF $2 \alpha$ protein levels and phosphorylation state during progression of iron deficiency (0-360 min). The levels of total eIF $2 \alpha$ and phosphorylated eIF2 $\alpha$ protein were determined by Western blot analyses using the anti-eIF2 $\alpha$ and anti-eIF2 $\alpha$-Ser51/52 antibodies, respectively. The pictures were cropped from two independent blots where the three biological replicates were included (Supplementary Fig. S1). (B) Quantification of the relative levels of eIF2 $\alpha-\mathrm{P} / \mathrm{eIF} 2 \alpha$ is represented in panel B. The average and standard deviation of three biological replicates is shown. Different letters over the bars indicate statistically significant differences ( $p$-value $<0.05)$. (C-F) Wild-type SUI2 (RS-86) $($ C,D) and SUI2-S51A mutant (RS-88) (E,F) strains were cultivated in SC $(+\mathrm{Fe})$ and SC with $100 \mu \mathrm{M}$ BPS $(-\mathrm{Fe})$ for 9 hours. Polysome analysis was performed as described in Fig. 1. Three biologically independent replicates were performed in each case, and a representative profile is shown. (G) The average $\mathrm{P} / \mathrm{M}$ ratio is represented with its corresponding standard deviation. Different letters over the bars indicate statistically significant differences ( $p$-value $<0.05)$.

kinase Gcn2 plays a role in the repression of global translation caused by iron depletion. It should be noted that the role of $\mathrm{Gcn} 2$ in translational repression seems to be transient since, upon a longer exposure to iron deficiency, the improvement of translation of a $g c n 2 \Delta$ mutant disappears and translation is inhibited to the same extent as in a wild-type strain (Supplementary Fig. S4). Taking all these results together, we conclude that the Gcn2-eIF $2 \alpha$ pathway is involved in the repression of global translation under iron starvation. 
A

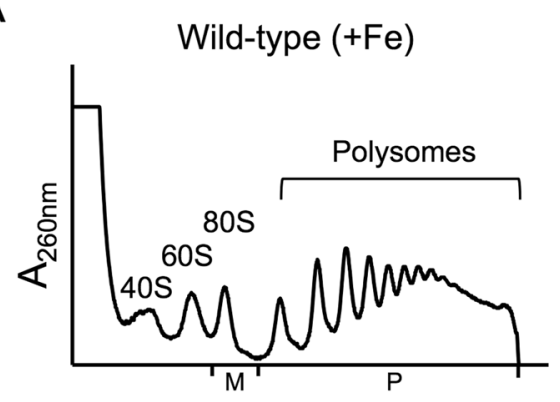

[Sucrose]

C

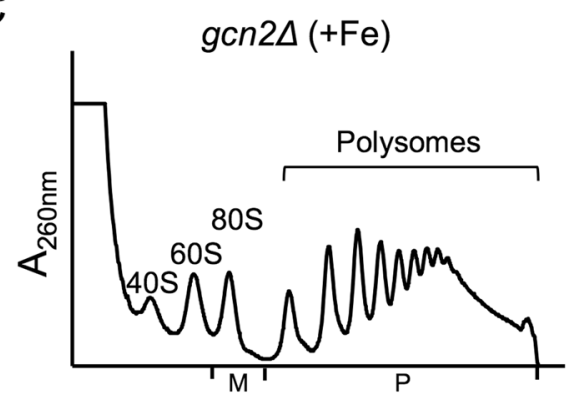

[Sucrose]

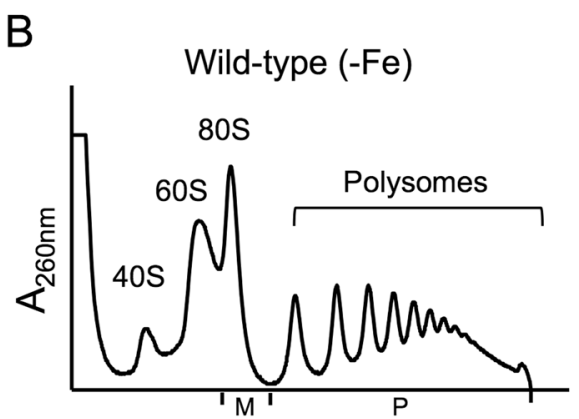

[Sucrose]

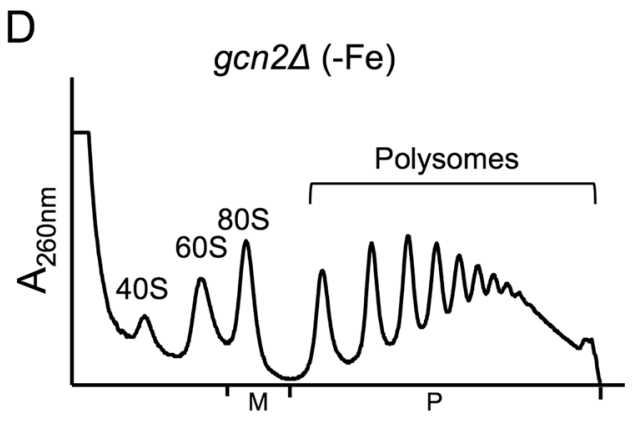

[Sucrose]

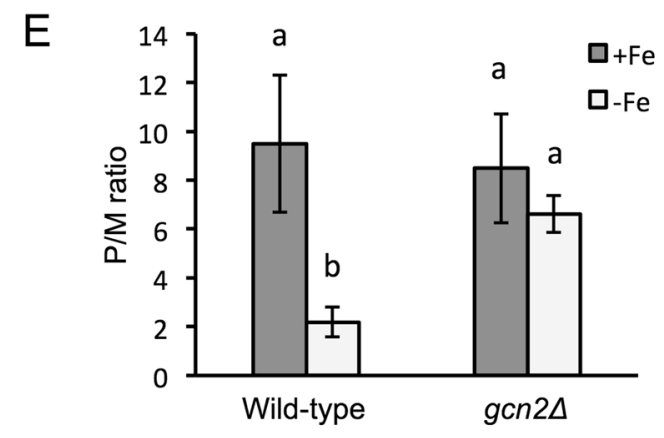

Figure 4. GCN2 deletion suppresses translation inhibition during the growth in iron scarcity. Wild-type BY4741 (A,B) and $g c n 2 \Delta(\mathbf{C , D})$ strains were cultivated in SC $(+\mathrm{Fe})$ and SC with $100 \mu \mathrm{M}$ BPS $(-\mathrm{Fe})$ for 9 hours. Polysome analysis was performed as described in Fig. 1. At least three biologically independent replicates were performed in each case, and a representative profile is shown. (E) The average P/M ratio is represented with its corresponding standard deviation. Different letters over the bars indicate statistically significant differences $(p-$ value $<0.05)$.

The repression of translation under iron deficiency depends on Gon1. The activation of Gcn2 under amino acid starvation conditions occurs through the binding of uncharged tRNAs to its histidyl-tRNA synthetase (HisRS)-like domain, a process that requires the Gcn $1-G c n 20$ protein complex ${ }^{25,30}$. To investigate whether the activation of Gcn 2 kinase in response to iron deficiency was mediated by this mechanism, we analyzed the polysome profile of wild-type and $g c n 1 \Delta$ cells under iron-deficient conditions. Similarly to the $g c n 2 \Delta$ mutant, $g c n 1 \Delta$ cells exhibited a grow defect in 3-AT-containing media and a defect in eIF2 $\alpha$ phosphorylation (Supplementary Figs. S2 and S3). Whereas there were no differences in the P/M ratio between wild-type and gcn $1 \Delta$ mutant cells under iron sufficiency (Fig. 5A,C,E), the translation inhibition that occurred on the wild-type strain under iron deficiency (Fig. 5B,E) was partially relieved in the gcn $1 \Delta$ mutant (Fig. 5D,E). These results indicate that Gcn 1 is required for the observed translation inhibition, and suggest that the mechanism that mediates Gcn 2 activation under iron deficiency involves the detection of uncharged tRNAs.

\section{Discussion}

Global repression of protein synthesis is one of the first responses of both prokaryotic and eukaryotic cells to nutritional deficiencies such as glucose or amino acid limitation, and to exposure to stress conditions like heat shock, osmotic and oxidative stress ${ }^{13-15,17,18}$. Opposite to glucose starvation and other nutritional stresses characterized by a rapid arrest of mRNA translation, iron deficiency is a nutritional disorder which effects are not immediate. At the initial stages of iron limitation, yeast cells activate several transcriptional and post-transcriptional 
A

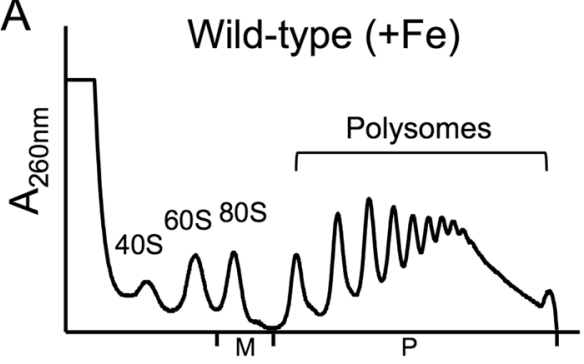

[Sucrose]

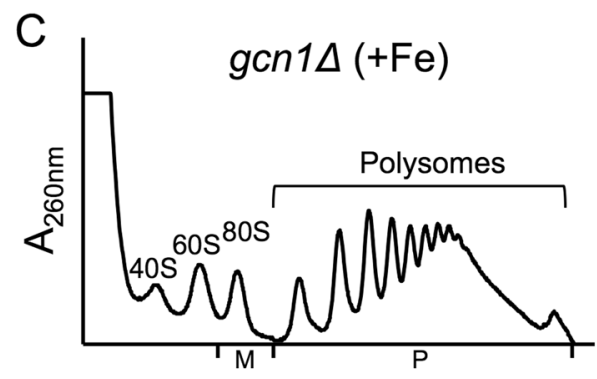

[Sucrose]
B

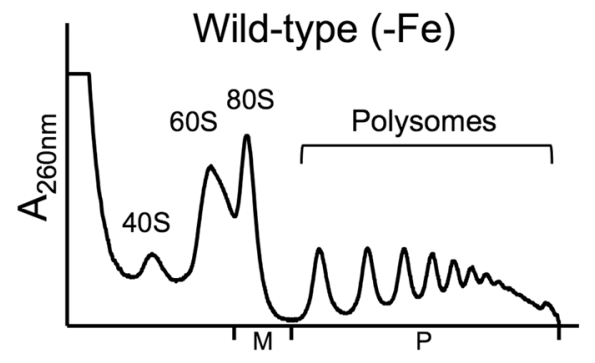

[Sucrose]

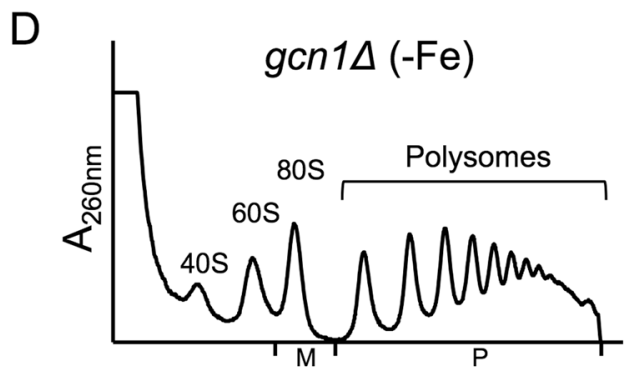

[Sucrose]

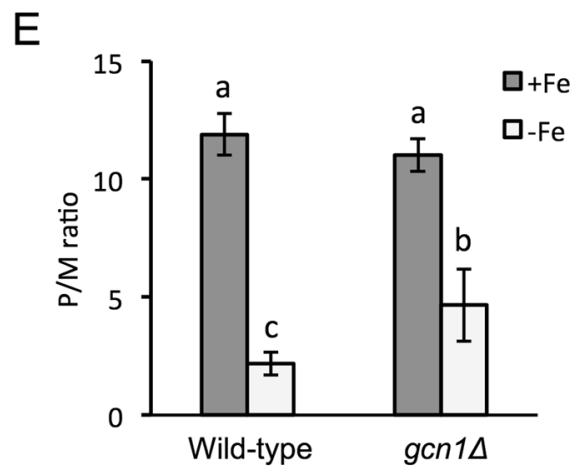

Figure 5. The deletion of GCN1 improves translation during the growth in iron scarcity. Wild-type BY4741 $(\mathbf{A}, \mathbf{B})$ and $g c n 1 \Delta(\mathbf{C}, \mathbf{D})$ strains were cultivated in SC $(+\mathrm{Fe})(\mathbf{A}, \mathbf{C})$ and SC with $100 \mu \mathrm{M}$ BPS $(-\mathrm{Fe})$ for 9 hours. (B,D) Polysome analysis was performed as described in Fig. 1. At least three biologically independent replicates were performed in each case, and a representative profile is shown. (E) The average P/M ratio is represented with its corresponding standard deviation. Different letters over the bars indicate statistically significant differences $(p$-value $<0.05)$.

regulatory mechanisms that promote the entrance of the scarce extracellular iron, the mobilization of intracellular iron stores, and the remodeling of iron metabolism in order to prioritize essential iron-dependent processes versus dispensable ones ${ }^{6,8,33}$. Therefore, no dramatic arrest of protein synthesis has been described after exposure to iron deficiency, which has been supported by the robust homeostasis of amino acid levels ${ }^{34,35}$. However, essential proteins involved in translation and ribosome recycling, such as Rli1, and multiple enzymes within various amino acid biosynthesis pathways are iron-containing proteins. Therefore, we hypothesized that upon longer iron deficiency periods, cells would not be able to maintain essential iron-dependent processes, and consequently translation would be affected. This prompted us to analyze whether a significant global arrest of translation was taking place after long exposure of yeast cells to iron limitation. For these experiments, we always maintained yeast cells at low cell densities, with an $\mathrm{OD}_{600}$ below or around 1.0, a condition at which cells not exposed to iron depletion maintained high translation levels. Under these conditions, we observed that mRNA translation only slightly decreased during a short-term exposure to iron scarcity (3 hours), while a more severe iron deficiency condition (6 hours) induced a dramatic repression of global translation (Fig. 1). Importantly this translational inhibition was not observed in a parallel culture grown for 6 hours under iron sufficiency with a similar $\mathrm{OD}_{600}$ (Fig. 1B,E), suggesting that the global translational arrest is specific of iron deficiency.

While translation is globally arrested in response to many stresses, eukaryotic cells keep or induce the translation of specific stress-responsive mRNAs, in order to adequately respond to a particular hazardous situation ${ }^{36-39}$. We have shown that this is also the case under iron limiting conditions, where different types of transcripts undergo distinct fates, according to their position in polyribosome profiles. While mRNAs encoding for ribosomal proteins shift towards the monosomal fractions, which is consistent with translation inhibition, the profile 
of the housekeeping actin gene is only slightly affected, and the uORF-containing GCN4 transcript shifts towards the polysomal fractions (Fig. 2). Some of the mechanisms by which the recruitment of ribosomes to selective stress-responsive mRNAs happens in a general translational inhibition scenario have been elucidated, and include the presence of Internal Ribosome Entry Sites (IRES), which allow those mRNAs to skip cap-dependent translation, and the presence of uORFs in the $5^{\prime}$-UTR, which in the case of GCN4 increase its translation when general translation initiation is inhibited due to low eIF2 ternary complex levels ${ }^{31,40,41}$. Our results support that the translation of GCN4 could be regulated through its uORFs when iron is scarce. However, the observed shift toward the polysomal fractions does not fully correlate with a parallel increase in GCN4-lacZ expression (Fig. 2E). These results suggest that the translation of specific transcripts under iron limiting conditions depends on additional mechanisms, which require further studies.

One of the principal mechanisms that control translation during stress conditions is the phosphorylation of serine 51 in eIF2 $\alpha$, which turns eIF2-GDP into a competitive inhibitor of eIF2B and decreases the assembly of ternary complexes. The augmented $80 \mathrm{~S}$ peak and the increased translation of GCN4 that we observed under iron deficiency were indicative of an arrest at initiation, which is the step of translation regulated by eIF2. We have demonstrated that eIF $2 \alpha$ is increasingly phosphorylated under iron deficiency (Fig. 3A,B), and that this phosphorylation is required for the global arrest of translation that occurs in response to iron scarcity (Fig. 3C-G). Gcn2 is the sole kinase that phosphorylates the alpha subunit of eIF2 in S. cerevisiae. Consistent with this, a gcn $2 \Delta$ mutant did not show the eIF2 $\alpha$ phosphorylation and the global inhibition of translation displayed by the wild-type strain upon iron deficiency (Supplementary Fig. S3 and Fig. 4). Therefore, we conclude that phosphorylation of eIF2 $\alpha$ by $\mathrm{Gcn} 2$ is necessary for this translational arrest. However, when the iron deficiency persists for a longer period, other mechanisms contribute to translational inhibition since no differences in the polysome profile are observed between $g c n 2 \Delta$ and wild-type cells after 12 hours of iron depletion (Supplementary Fig. S4).

The role played by Gcn2-dependent phosphorylation of eIF $2 \alpha$ in the global inhibition of translation that yeast cells undergo in iron deficiency seems to be conserved. In mammals, iron deficiency limits heme synthesis, which activates heme-regulated eIF2 $\alpha$ kinase (HRI), one of the four mammalian eIF2 $\alpha$ kinases that phosphorylate eIF2 $\alpha$. This causes a decrease in bulk translation, down-regulating the synthesis of globins while increasing the translation of specific transcripts, such as $\mathrm{HbF}$ (coding for fetal hemoglobin) in certain anemias ${ }^{42-45}$. The targeted deletion of HRI causes an improvement of global translation, similar to that observed for the deletion of yeast GCN2, which increases globin translation ${ }^{42}$. In addition to HRI, mammalian cells possess three other eIF $2 \alpha$ kinases, which are expressed in different tissues and activated by specific physiological stresses, namely GCN2, activated by nutrient deprivation; PKR, which responds to viral infection; and PERK, activated by endoplasmic reticulum (ER) stress ${ }^{28,46-50}$. The phosphorylation of eIF $2 \alpha$ by any of these stress-activated kinases induces a stress-resistant state that results from the attenuation of global translation, diverting amino acids to other metabolic necessities, while selectively enhancing translation of ATF4, the mammalian GCN4 counterpart. This stress response pathway, of which eIF2 $\alpha$ is the central node, has been termed the Integrated Stress Response (ISR) ${ }^{51,52}$, and was initially outlined in yeast as the General Amino Acid Control (GAAC), a response to amino acid depletion that induced the expression of amino acid biosynthetic genes ${ }^{53}$. Since then, Gcn 2 has been described to be activated by a wide variety of stress conditions in yeast, including purine starvation, glucose limitation, ER stress, ethanol, heat shock, salt stress, acid exposure, and oxidative stress ${ }^{17,54-58}$. The present study adds iron starvation to the list of conditions that activate Gcn2. Thus, the ISR could be considered a stress response conserved from yeast to mammals.

The activation of Gcn 2 by amino acid starvation requires both the interaction with the Gcn1-Gcn20 complex and its binding to uncharged tRNAs through its histidyl-tRNA synthetase (HisRS)-like domain ${ }^{25,30}$. The current model proposes that Gcn 1 binds to the ribosome, close to the A site, mediating the transfer of the uncharged tRNA to $G \mathrm{cn} 2^{26}$. In yeast, the activation of Gcn2 under other stresses, such as oxidative stress, depends on Gcn 1 and Gcn 20 regulatory factors, and therefore on the sensing of tRNA levels ${ }^{17}$. Gcn 20 is not essential for Gcn2-mediated GCN4 translational control under glucose limitation, but it is required for full repression ${ }^{55}$. By performing polysomal profiles of $g c n 1 \Delta$ mutant cells, we show that Gcn1 is important for the repression of protein synthesis under iron deficiency (Fig. 5). Therefore, we conclude that the sensing of uncharged tRNAs is the main mechanism that activates $\mathrm{Gcn} 2$ in response to iron deficiency. This seems to be the main way of activation of Gcn2 in S. cerevisiae, fission yeast, and mammals, in response to a wide range of stresses, even those where no obvious accumulation of uncharged tRNAs occurs, such as UV treatment. This common mechanism of Gcn 2 activation by different types of stress underscores the relevance of the ISR as a general and conserved way of eukaryotic cells to cope with stress and nutrient limitation ${ }^{52}$. In spite of many common features, the concrete ISR-mediated mechanisms that determine cell fate in response to each stress are only starting to be deciphered in mammalian cells, and therefore, understanding which specific mechanisms are activated downstream of Gcn2-eIF2 $\alpha-G c n 4$ in iron scarcity remains an intriguing challenge that needs to be addressed.

\section{Methods}

Yeast strains and growth conditions. The S. cerevisiae strains used in this study are listed in the Supplementary Table S1. Yeast cells were cultivated in synthetic media to exponential phase as indicated. To induce iron deficiency, $100 \mu \mathrm{M}$ of the $\mathrm{Fe}^{2+}$-specific chelator bathophenanthroline disulfonic acid (BPS; Sigma), whereas 3-aminotriazol (3-AT) treatment was used to induce amino acid starvation. Aliquots were isolated at the indicated times for further analyses.

Polyribosome profiles analyses. Yeast cells were cultivated to exponential phase in SC or SC $+100 \mu \mathrm{M}$ BPS, and aliquots were taken at the indicated times. Polyribosome profiles analyses were performed as previously described ${ }^{8,38}$. For the RNA analyses of the polysomal fractions, lys and spo mRNAs from Bacillus subtilis were added to $200 \mu \mathrm{L}$ of each fraction before extraction at a concentration of $3 \mathrm{ng} / \mu \mathrm{L}$. RNA was extracted with 
the SpeedTools Total RNA Extraction kit (Biotools B\&M Labs) and a DNAse treatment was performed after the RNA elution step. Specific primer pairs were used to analyze mRNAs by RT-qPCR (Supplementary Table S2), and values were represented as a percentage of the total. All the values were normalized with the spiked-in mRNA levels of $B$. subtilis lys and spo. At least three biological replicates were performed for each polyribosome profile, and a representative profile is shown. The average polysomes/monosome $80 \mathrm{~S}(\mathrm{P} / \mathrm{M})$ ratio was represented with its corresponding standard deviation.

GCN4-lacZ expression assays. Cells transformed with the GCN4-lacZ reporter plasmid (p180) ${ }^{27}$ were cultivated overnight in synthetic minimal medium (SD), and then diluted and reinoculated in SD $(+\mathrm{Fe})$ or $\mathrm{SD}+100 \mu \mathrm{M}$ BPS $(-\mathrm{Fe})$ for 6 hours, and SD $+30 \mathrm{mM} 3$-AT for 5 hours. Cells were harvested and $ß$-galactosidase activity was measured in permeabilized cells as previously described ${ }^{59}$.

Protein analyses. Yeast proteins were extracted and analyzed by Western blot as previously reported ${ }^{8}$. The primary antibodies used in this study included anti-eIF2 $\alpha$ (from John M. Zaborske) and anti-eIF2 $\alpha$-Ser51/Ser52 (Cell Signaling Technology). Images were obtained with an ImageQuant LAS 4000 mini biomolecular imager, and specific signals were quantified with ImageQuant TL analysis software (GE Healthcare Life Sciences).

Statistical analyses. The data and error bars represent the average and standard deviations of at least three independent biological samples. Two tailed t-student tests were applied to evaluate statistical significance. When the statistical test indicated that two means are significantly different from each other $(p$-value $<0.05)$, the bars were labeled with different letters.

Received: 26 July 2019; Accepted: 16 December 2019;

Published online: 14 January 2020

\section{References}

1. Duhutrel, P. et al. Iron sources used by the nonpathogenic lactic acid bacterium Lactobacillus sakei as revealed by electron energy loss spectroscopy and secondary-ion mass spectrometry. Appl. Environ. Microbiol. 76, 560-565 (2010).

2. Posey, J. E. \& Gherardini, F. C. Lack of a role for iron in the Lyme disease pathogen. Science 288, 1651-1653 (2000).

3. Salvail, H. \& Masse, E. Regulating iron storage and metabolism with RNA: an overview of posttranscriptional controls of intracellular iron homeostasis. Wiley Interdiscip. Rev. RNA 3, 26-36 (2012).

4. Carrier, M. C., Bourassa, J. S. \& Masse, E. Cellular Homeostasis: A Small RNA at the Crossroads of Iron and Photosynthesis. Curr. Biol. 27, R38-R383 (2017).

5. Muckenthaler, M. U., Galy, B. \& Hentze, M. W. Systemic iron homeostasis and the iron-responsive element/iron-regulatory protein (IRE/IRP) regulatory network. Annu. Rev. Nutr. 28, 197-213 (2008).

6. Puig, S., Askeland, E. \& Thiele, D. J. Coordinated remodeling of cellular metabolism during iron deficiency through targeted mRNA degradation. Cell 120, 99-110 (2005).

7. Pedro-Segura, E. et al. The Cth2 ARE-binding protein recruits the Dhh1 helicase to promote the decay of succinate dehydrogenase SDH4 mRNA in response to iron deficiency. J. Biol. Chem. 283, 28527-28535 (2008).

8. Ramos-Alonso, L. et al. Yeast Cth2 protein represses the translation of ARE-containing mRNAs in response to iron deficiency. PLoS Genet. 14, e1007476 (2018)

9. Bayeva, M. et al. mTOR regulates cellular iron homeostasis through tristetraprolin. Cell. Metab. 16, 645-657 (2012).

10. Sato, T. et al. mRNA-binding protein tristetraprolin is essential for cardiac response to iron deficiency by regulating mitochondrial function. Proc. Natl. Acad. Sci. USA 115, E629-E6300 (2018).

11. Buttgereit, F. \& Brand, M. D. A hierarchy of ATP-consuming processes in mammalian cells. Biochem. J. 312(Pt 1), 163-167 (1995).

12. Russell, J. B. \& Cook, G. M. Energetics of bacterial growth: balance of anabolic and catabolic reactions. Microbiol. Rev. 59, 48-62 (1995).

13. Martinez-Pastor, M. T. \& Estruch, F. Sudden depletion of carbon source blocks translation, but not transcription, in the yeast Saccharomyces cerevisiae. FEBS Lett. 390, 319-322 (1996).

14. Ashe, M. P., De Long, S. K. \& Sachs, A. B. Glucose depletion rapidly inhibits translation initiation in yeast. Mol. Biol. Cell 11, 833-848 (2000).

15. Starosta, A. L., Lassak, J., Jung, K. \& Wilson, D. N. The bacterial translation stress response. FEMS Microbiol. Rev. 38, 1172-1201 (2014).

16. Hinnebusch, A. G. Translational regulation of GCN4 and the general amino acid control of yeast. Annu. Rev. Microbiol. 59, 407-450 (2005).

17. Shenton, D. et al. Global translational responses to oxidative stress impact upon multiple levels of protein synthesis. J. Biol. Chem. 281, 29011-29021 (2006).

18. Yamamoto, Y. \& Izawa, S. Adaptive response in stress granule formation and bulk translational repression upon a combined stress of mild heat shock and mild ethanol stress in yeast. Genes Cells 18, 974-984 (2013).

19. Dever, T. E. et al. Phosphorylation of initiation factor 2 alpha by protein kinase GCN2 mediates gene-specific translational control of GCN4 in yeast. Cell 68, 585-596 (1992).

20. Gebauer, F. \& Hentze, M. W. Molecular mechanisms of translational control. Nat. Rev. Mol. Cell Biol. 5, 827-835 (2004).

21. Jackson, R. J., Hellen, C. U. \& Pestova, T. V. The mechanism of eukaryotic translation initiation and principles of its regulation. Nat. Rev. Mol. Cell Biol. 11, 113-127 (2010).

22. Sonenberg, N. \& Hinnebusch, A. G. Regulation of translation initiation in eukaryotes: mechanisms and biological targets. Cell 136, $731-745$ (2009).

23. Hershey, J. W., Sonenberg, N. \& Mathews, M. B. Principles of translational control: an overview. Cold Spring Harb Perspect. Biol., 4, https://doi.org/10.1101/cshperspect.a011528 (2012).

24. Dever, T. E., Kinzy, T. G. \& Pavitt, G. D. Mechanism and Regulation of Protein Synthesis in Saccharomyces cerevisiae. Genetics 203, 65-107 (2016).

25. Garcia-Barrio, M., Dong, J., Ufano, S. \& Hinnebusch, A. G. Association of GCN1-GCN20 regulatory complex with the N-terminus of eIF2alpha kinase GCN2 is required for GCN2 activation. EMBO J. 19, 1887-1899 (2000).

26. Marton, M. J., Vazquez de Aldana, C. R., Qiu, H., Chakraburtty, K. \& Hinnebusch, A. G. Evidence that GCN1 and GCN20, translational regulators of GCN4, function on elongating ribosomes in activation of eIF2alpha kinase GCN2. Mol. Cell. Biol. 17, 4474-4489 (1997). 
27. Hinnebusch, A. G. A hierarchy of trans-acting factors modulates translation of an activator of amino acid biosynthetic genes in Saccharomyces cerevisiae. Mol. Cell. Biol. 5, 2349-2360 (1985).

28. Dever, T. E. Translation initiation: adept at adapting. Trends Biochem. Sci. 24, 398-403 (1999).

29. Castilho, B. A. et al. Keeping the eIF2 alpha kinase Gcn2 in check. Biochim. Biophys. Acta 1843, 1948-1968 (2014).

30. Anda, S., Zach, R. \& Grallert, B. Activation of Gcn2 in response to different stresses. PLoS One 12, e0182143 (2017).

31. Hinnebusch, A. G. Translational control of GCN4: an in vivo barometer of initiation-factor activity. Trends Biochem. Sci. 19, 409-414 (1994).

32. Caballero-Molada, M. et al. The Gcn2-eIF2alpha pathway connects iron and amino acid homeostasis in Saccharomyces cerevisiae. Biochem. J. 475, 1523-1534 (2018).

33. Martinez-Pastor, M. T., de Llanos, R., Romero, A. M. \& Puig, S. Post-transcriptional regulation of iron homeostasis in Saccharomyces cerevisiae. Int. J. Mol. Sci. 14, 15785-15809 (2013).

34. Shakoury-Elizeh, M. et al. Metabolic response to iron deficiency in Saccharomyces cerevisiae. J. Biol. Chem. 285, 14823-14833 (2010).

35. Philpott, C. C., Leidgens, S. \& Frey, A. G. Metabolic remodeling in iron-deficient fungi. Biochim. Biophys. Acta 1823, 1509-1520 (2012).

36. Melamed, D., Pnueli, L. \& Arava, Y. Yeast translational response to high salinity: global analysis reveals regulation at multiple levels. RNA 14, 1337-1351 (2008)

37. Warringer, J., Hult, M., Regot, S., Posas, F. \& Sunnerhagen, P. The HOG pathway dictates the short-term translational response after hyperosmotic shock. Mol. Biol. Cell 21, 3080-3092 (2010).

38. Garre, E. et al. Yeast mRNA cap-binding protein $\mathrm{Cbc1/Sto1}$ is necessary for the rapid reprogramming of translation after hyperosmotic shock. Mol. Biol. Cell 23, 137-150 (2012).

39. Garre, E., Pelechano, V., Sanchez Del Pino, M., Alepuz, P. \& Sunnerhagen, P. The Lsm1-7/Pat1 complex binds to stress-activated mRNAs and modulates the response to hyperosmotic shock. PLoS Genet. 14, e1007563 (2018).

40. Spriggs, K. A., Stoneley, M., Bushell, M. \& Willis, A. E. Re-programming of translation following cell stress allows IRES-mediated translation to predominate. Biol. Cell 100, 27-38 (2008).

41. Hinnebusch, A. G., Ivanov, I. P. \& Sonenberg, N. Translational control by 5 '-untranslated regions of eukaryotic mRNAs. Science 352, 1413-1416 (2016).

42. Han, A. P. et al. Heme-regulated eIF2alpha kinase (HRI) is required for translational regulation and survival of erythroid precursors in iron deficiency. EMBO J. 20, 6909-6918 (2001).

43. Hahn, C. K. \& Lowrey, C. H. Eukaryotic initiation factor 2alpha phosphorylation mediates fetal hemoglobin induction through a post-transcriptional mechanism. Blood 122, 477-485 (2013).

44. Hahn, C. K. \& Lowrey, C. H. Induction of fetal hemoglobin through enhanced translation efficiency of gamma-globin mRNA. Blood 124, 2730-2734 (2014).

45. Zhang, S. et al. HRI coordinates translation necessary for protein homeostasis and mitochondrial function in erythropoiesis. Elife, 8, https://doi.org/10.7554/eLife.46976 (2019).

46. Hinnebusch, A. G. The eIF-2 alpha kinases: regulators of protein synthesis in starvation and stress. Semin. Cell Biol. 5, 417-426 (1994).

47. Sood, R., Porter, A. C., Olsen, D. A., Cavener, D. R. \& Wek, R. C. A mammalian homologue of GCN2 protein kinase important for translational control by phosphorylation of eukaryotic initiation factor-2alpha. Genetics 154, 787-801 (2000).

48. Clemens, M. J. PKR-a protein kinase regulated by double-stranded RNA. Int. J. Biochem. Cell Biol. 29, 945-949 (1997).

49. Shi, Y. et al. Identification and characterization of pancreatic eukaryotic initiation factor 2 alpha-subunit kinase, PEK, involved in translational control. Mol. Cell. Biol. 18, 7499-7509 (1998).

50. Harding, H. P., Zhang, Y. \& Ron, D. Protein translation and folding are coupled by an endoplasmic-reticulum-resident kinase. Nature 397, 271-274 (1999).

51. Harding, H. P. et al. An integrated stress response regulates amino acid metabolism and resistance to oxidative stress. Mol. Cell 11, 619-633 (2003).

52. Pakos-Zebrucka, K. et al. The integrated stress response. EMBO Rep. 17, 1374-1395 (2016).

53. Hinnebusch, A. G. \& Fink, G. R. Positive regulation in the general amino acid control of Saccharomyces cerevisiae. Proc. Natl. Acad. Sci. USA 80, 5374-5378 (1983).

54. Rolfes, R. J. \& Hinnebusch, A. G. Translation of the yeast transcriptional activator GCN4 is stimulated by purine limitation: implications for activation of the protein kinase GCN2. Mol. Cell. Biol. 13, 5099-5111 (1993).

55. Yang, R., Wek, S. A. \& Wek, R. C. Glucose limitation induces GCN4 translation by activation of Gen2 protein kinase. Mol. Cell. Biol. 20, 2706-2717 (2000).

56. Deloche, O., de la Cruz, J., Kressler, D., Doere, M. \& Linder, P. A membrane transport defect leads to a rapid attenuation of translation initiation in Saccharomyces cerevisiae. Mol. Cell 13, 357-366 (2004).

57. Goossens, A., Dever, T. E., Pascual-Ahuir, A. \& Serrano, R. The protein kinase Gcn2p mediates sodium toxicity in yeast. J. Biol. Chem. 276, 30753-30760 (2001).

58. Gallinetti, J., Harputlugil, E. \& Mitchell, J. R. Amino acid sensing in dietary-restriction-mediated longevity: roles of signaltransducing kinases GCN2 and TOR. Biochem. J. 449, 1-10 (2013).

59. Liu, X. D., Morano, K. A. \& Thiele, D. J. The yeast Hsp110 family member, Sse1, is an Hsp90 cochaperone. J. Biol. Chem. 274, 26654-26660 (1999).

\section{Acknowledgements}

We thank the members of the "Iron Homeostasis" laboratory for scientific comments and technical assistance. We are also grateful to Drs. Fred Cross, Emilia Matallana, Ramón Serrano, Alan G. Hinnebusch, Mercedes Tamame, Paqui Rández-Gil, John M. Zaborske, and José Marcos for providing yeast strains, plasmids and antibodies used in this study. This work was supported by predoctoral contracts from the Spanish Ministry of Science, Innovation and Universities (MICINN) to AMR and LRA, and European Union Funds (FEDER) and MICINN grants BIO2017-87828-C2-1-P to SP and BFU2016-77728-C3-3-P to PA.

\section{Author contributions}

A.M.R.: Investigation, Writing - review and editing, visualization; Analysis and interpretation of data. L.R.-A.: Investigation, Writing - review and editing, visualization; Analysis and interpretation of data. P.A.: Resources, Writing - review and editing, Funding acquisition, Analysis and interpretation of data. S.P.: Conceptualization, Resources, Writing - original draft, Funding acquisition, Project administration, visualization. M.T.M.-P.: Conceptualization, Writing - original draft, Project administration, visualization. 


\section{Competing interests}

The authors declare no competing interests.

\section{Additional information}

Supplementary information is available for this paper at https://doi.org/10.1038/s41598-019-57132-0.

Correspondence and requests for materials should be addressed to S.P. or M.T.M.-P.

Reprints and permissions information is available at www.nature.com/reprints.

Publisher's note Springer Nature remains neutral with regard to jurisdictional claims in published maps and institutional affiliations.

(c) (i) Open Access This article is licensed under a Creative Commons Attribution 4.0 International License, which permits use, sharing, adaptation, distribution and reproduction in any medium or format, as long as you give appropriate credit to the original author(s) and the source, provide a link to the Creative Commons license, and indicate if changes were made. The images or other third party material in this article are included in the article's Creative Commons license, unless indicated otherwise in a credit line to the material. If material is not included in the article's Creative Commons license and your intended use is not permitted by statutory regulation or exceeds the permitted use, you will need to obtain permission directly from the copyright holder. To view a copy of this license, visit http://creativecommons.org/licenses/by/4.0/.

(C) The Author(s) 2020 